\title{
Strategy of Wireless Network Resource Management Based on Cooperative Cognition
}

\author{
Wuxue Jiang ${ }^{1, a}$, Shi Wang ${ }^{1, b}$ \\ ${ }^{1}$ Department of Computer Engineering, Dongguan Polytechnic, Dongguan 523808, Guangdong, China \\ ajiangwx@dgpt.edu.cn, b8668816@qq.com
}

\begin{abstract}
In recent years, the wireless network technology has made great progress, including WiFi, WiMax, Bluetooth, ultra wide-band, $4 \mathrm{G}$ and so on. In order to reduce the interference between different wireless technologies and improve the utilization of wireless resources, this paper studies the unique characteristics of multi-radio wireless networks, proposing collaborative and cognitive networking concept. The strategy aims to ensure the auto organize wireless network device to obtain the best connection characteristics, and to improve the utilization efficiency of scarce radio resources, simultaneously. The simulation results show that the proposed strategy is feasible which can improve the efficiency.
\end{abstract}

Keywords: wireless network, resource management, cognitive strategy

\section{Introduction}

In recent years, wireless network technology has made great progress, including WiFi, WiMax, Bluetooth, ultra wide-band, $4 \mathrm{G}$ and so on. These technologies will coexist to form a multi- radio environment to provide different service capabilities for various applications of users. With the rapid growth of wireless devices, especially the increase of multi-radio / multi-band devices, people are generally concerned about the problem of connecting to the best communication network at any time. Therefore, supporting the research of multiple radio networks is one of the hottest areas at present. There are many challenges in technology: due to the shared characteristics of the wireless media, the adjacent devices will interfere with each other, which can result in significant degradation of the system performance. In order to reduce the interference between different wireless technologies, the existing frequency band allocation strategy divides the spectrum into non overlapping and fixed frequency bands, which are allocated to different wireless technologies or services, which can lead to a low radio resource utilization. At the same time, the control mechanisms at different levels in the wireless network have complex interactions. All these aspects lead to the extremely difficult problem of radio resource management. The goal of this study is to study on unique characteristics of multi-radio wireless networks, proposing CCN (collaborative and cognitive networking) concept, so as to ensure the auto organized wireless network device can obtain the best connection characteristics, so as to improve the utilization efficiency of scarce radio resources simultaneously.

The current theoretical research generally assumes that the ideal media access control (ideal distribution is achieved without conflict), assuming that a central controller has all the information, which makes the approximate algorithm they design can not be applied to the actual deployment. As for distributed algorithm, most of the related researches consider channel allocation and routing separately. Some articles also consider channel allocation and routing at the same time, but do not relate them. In this framework, the key grid has access points connected by wireless network. This scheme is only available in a tree - like topology of wireless networks. An effective way to efficiently use the energy is to reduce the transmission power as much as possible. However, in a network that supports multiple rates, the reduction of transmission power may result in a reduction in the transmission rate. Therefore, power control and rate adaptive need to be considered simultaneously. G. Holland, N. Vaidya and P. Bahl proposed a rate of RBAR (Receiver-Based 
Auto-Rate ), through RBAR, the adjustment of rate is controlled by the receiver rather than the sender, sending request / clearing the sending request, which is used to detect the channel condition and adjust the rate according to the situation. B. Sadeghi proposed OAR (Opportunistic Auto Rate). The main idea of OAR is to send multiple consecutive packets with opportunistic transmission as long as the channel is in good condition. However, the aim of the above research work is to optimize the throughput or delay performance of the data flow, and not to deal with the problem of energy efficiency.

\section{The Basic Architecture and Process of System}

The commonly recognized SDN architecture is a network architecture based on OpenFlow protocol proposed by ONF, which is also a reference standard for many other frameworks. SDN can integrate hardware and software of traditional network gradually, transforming them into the bottom and top of the separate controlling architecture, network hardware devices become simpler and more intelligent. While the upper intelligent service strategy and management layer will provide a way of software, such as open API interface, developers can write all kinds of application through the interface. SDN decoupled the control from the data forwarding function in the traditional network devices. SDN can be divided into application layer, control layer and infrastructure layer. SDN framework is able to control the entire network status, by controlling the perception layer and user specific cellular network heterogeneous changes in demand for QoE, and then through the support of OpenFlow protocol interface to control the communication infrastructure, meanwhile the interface is open to users according to the needs of businesses, operators can collect data the needed information, and provide different services according to the user demand, so as to realize the automation of network control.

In traditional networks, the control plane and data plane exist simultaneously in the network nodes, that is to say, each node has its own data and control plane. The control plane in the traditional network is responsible for configuring nodes and assigning appropriate paths to data streams through network programming. After the path is determined, the data stream is pushed down to the data plane, and the data is forwarded in the hardware based on the information of the control plane. In this traditional network architecture, once the data flow management policy (forwarding policy) is decided, the only way to adjust the policy is to change the configuration of the device. However, the network of isomerization is more and more obvious, the type of business is increased, under the condition that the user demands are changed,this type of data can limit the change of network strategy operators due to traffic demand changes in the flexible operation, at the same time, in the era of Big Data, the number of users' connecting devices is increased, for the difference of the needs of users, any one operator, or any kind of network architecture can meet the needs of requirements.

Based on the actual needs of users and operators based on change and service, SDN as a new network architecture can well meet the requirements of flexibility, simplicity, shortcut, low cost and scalability and so on. The control plane is removed from a single network node and a separate SDN centralized controller can be set up. The infrastructure layer SDN switches are controlled by the network operating system (Network Operating System, NOS), supporting OpenFlow protocol data / control plane API to collect data, manipulating the data forwarding plane, so as to provide an abstract network topology model to SDN controller. Therefore, SDN controller can take advantage of network. In M3WN, channel allocation and routing are closely related, on one hand, the channel allocation determines the radio connectivity, because between two radio, only when they are in a common channel can they communicate with each other, so the channel allocation can determine the topology of the network. Routing strategies are based on the topology of the network, so channel allocation has a direct impact on routing. On the other hand, in order to achieve better results, channel allocation should be dynamically adjusted according to the state of communication, 
and the amount of communication is determined by the routing algorithm. As a result, routing and channel allocation are tightly coupled.

The topology with six nodes, each node is represented by a small solid circle, the small soltorus can present the interface of $802.11 \mathrm{a}$, while the hollow ring can represent the interface of $802.11 \mathrm{~g}$, among the nodes, only the node N1 supports the radio of $802.11 \mathrm{a}$, for the rest of the nodes, each of them supports both the radio of 802.11 a and 802.11g, as is shown in Fig.1.
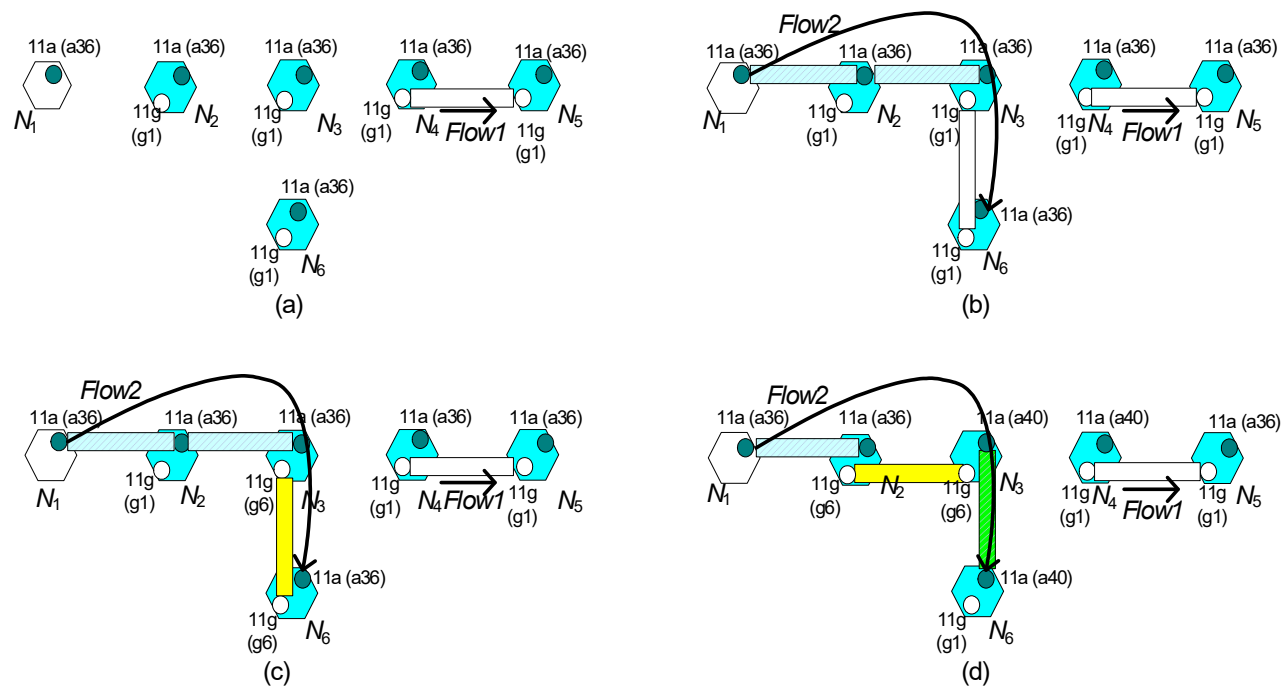

Fig.1 The Distribution of Channel and the Structure of Topology

\section{Algorithm and Improvement Strategy}

In sub-channel allocation, it is assumed that a sub-channel can only be assigned to a cooperative group at most to avoid interference between the cooperative groups during the signal process. The pseudo code for the sub-channel $r$ allocation process can be expressed as:

1) initialization

2) while $(\mathrm{i}<=\mathrm{N})$

while $(\mathrm{k}<=\mathrm{K})$

Assuming $\rho_{k n}=1$

Obtain $W_{n k}=h(p)$ according to the formula (1)

Assuming $\rho_{k n}=0, p^{*}=\arg \max W_{k n}$

Assuming $\rho_{k n}^{*}=1, \Omega_{K^{*}}{ }^{*}=\Omega_{K^{*}} \cup\{n\}, \rho_{k n}=0, \forall k \neq k^{*}$

Among them, the formula (1) is as follows:

$$
\rho_{k n} \in\{0,1\}, \sum_{k=1}^{K} \rho_{k n} \leq 1, \forall k, n
$$

Assuming that the power allocation matrix $p$ has been given, then the sub-channel assignment of home base station can be defined as: the sub-channel $l$ is allocated to the smallest $I_{u_{f}, I} / g_{f, l}$ user when the user's minimum business requirements are met and the channel usage allocation principle can be satisfied.

For each home base station $f$, the algorithm of sub-channel allocation can be shown in Table 1 .

Table 1 Algorithm of Channel Allocation

\begin{tabular}{l} 
Initialization: $\Omega_{u_{f}, l}=\varnothing, u \in U_{f}, v_{u f}^{r e q}, L, v_{u f}=0$ \\
for each $u \in U_{f}$ \\
while $U_{f} \neq \varnothing$ or $L \neq \varnothing$ \\
\hline
\end{tabular}


The best unused sub-channel pairing $\left(u, l^{*}\right)=\arg \min \left(I_{u_{f}, l} / g_{f, l}\right)$ for terminal user $\mathrm{u}$ is found from $\mathrm{L}$

Calculating the reachable rate $v_{u_{f}}=\Delta f \log \left(1+\gamma_{u_{f}, l}\right)$

if $\left(\left(v_{u_{f}} \geq v_{u_{f}}^{r e q}\right) \Omega_{u_{f}, l}=\Omega_{u_{f}, l}+l^{*}, U_{f}=U_{f}-u, L=L-l^{*}\right)$

end

end

\section{Scene of Application}

Ultra dense wireless networks can deploy ultra high density of various types of small base stations to meet the requirements of ultra high traffic and data traffic both indoor and outdoor. In November, 2017, HUAWEI conducted a ultra dense networking test. The purpose of this experiment is to measure the effectiveness of the ultra dense eNodeB station base station for network capacity enhancement, evaluate the performance of the network so as to ensure the user's experience. In complex radio environment, in order to provide a good network experience for large venues, compared with traditional mobile communication network, eNodeB small base stations have the features of flexible installation, low power consumption and low cost. Ultra dense wireless networks show high quality experience both inside and outside the place.

In densely populated laboratories or business office areas and other crowded hot spots. Due to the high density of access points of eNodeB stations and different load volume of different periods, it will cause serious interference on the same level, difficulties of resource allocation, as well as energy waste. Therefore, building a resource management verification platform, adopting SDN to realize the use of real-time monitoring and plug-in development of various strategies for the whole network eNodeB station resources, so as to realize the implementation of forwarding and control panel separation, which is the experiment target in this chapter. Let wireless communication return to "the last mile", narrowing the distance between users and antennas, increasing the rate and enhancing service coverage area, and implementing the protocol interface with SDN idea, so as to achieve the centralize management and flexible scheduling of signal resources.

\section{Application Simulation}

Each system module network mainly uses Socket to interact. Socket communication is based on TCP/IP (Transmission Control Protocol/Internet Protocol, TCP/IP) communication protocol, data format protocol adopts the south interface protocol Open SC. The hardware and software environment is as follows:

Developed Hardware Environment:

(1) hardware environment of e Node B small station:

Having LTE base station with programmable and supporting the south interface protocol;

Supporting 4G band of the mobile, working mode TD-LTE (Time Division Duplexing LTE), FDD-LTE (Frequency Division Duplexing LTE);

which can be configured for network and wireless and other parameters.

(2) PC hardware environment:

CPU:Inter (M) Core (TM) i7-6700

Memory: $8 \mathrm{G}$

Capacity of hard disk : $2 \mathrm{~T}$

Developed Software Environment:

(1) PC operating system: Window 10 Ultimate

(2) Development tools: Intellij idea 14.04, g++

(3) SDN open source framework: Floodlight

(4) Web server: Tomcat 8+JDK 1.8 
(5) Database tools: My Sq1 5.7.17

In system simulation, assuming the diameter of Macro site station can cover $500 \mathrm{~m}$, in the dense areas and dense commercial areas deploy several home base stations, the coverage of every home base station is $20 \mathrm{~m}$, the distance of each is $20-50$ meters. K-means algorithm can be adopted to cluster the home base station, users are randomly distributed in their respective areas, the number of Macro site station is 50 . The number of home base station is 2-10 people.

The relationship between the evolution of energy efficiency and the number of iterative times in a ultra dense wireless network. From the graph, we can see that with the increase of iteration times, the evolution of energy efficiency is gradually converging, and energy efficiency has reached the state of convergence within 25 iterations.

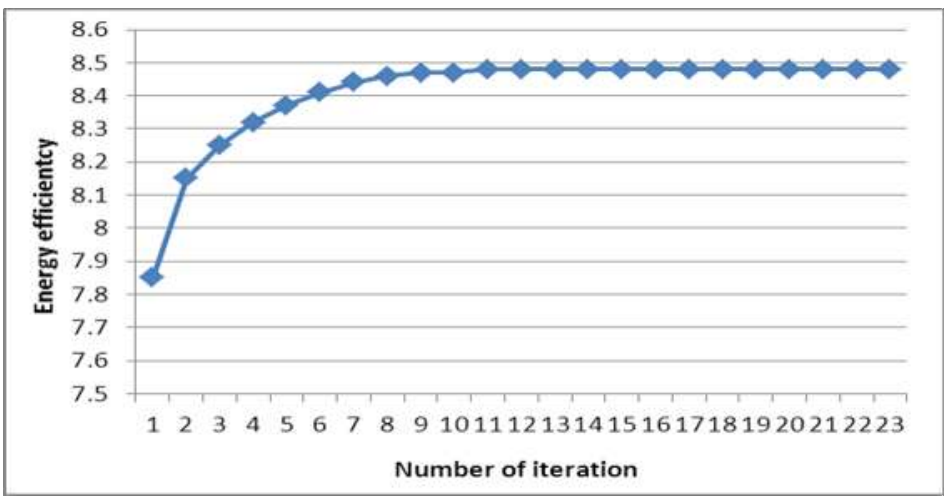

Fig.3 Relation between Evolution of Energy Efficiency and Number of Algorithm Iteration

Fig.2 describes the relationship between the average energy efficiency of each sub-channel and the number of terminal users. As you can see from the figure, with the number of terminal users increased, the average energy efficiency of the home base station users are also increased, which is due to the impact of multi-user diversity. In the sub-channel allocation process, the proposed algorithm can select one of the users with the highest signal-to-noise ratio from more terminal users to carry out sub-channel allocation.

\section{Conclusion}

In this paper, according to the super dense wireless cyber source allocation algorithm, mainly from the premise to reduce the interference from the two aspects of maximum throughput and energy efficiency as the goal to propose the resource allocation algorithm in two dimensions of power and sub channel, compared with the traditional resource allocation algorithm, it has certain advantages, but there are many aspects that still need further improvement.

\section{Acknowledgements}

This work was financially supported by the fund project of Dongguan Polytechnic (No.2017a06), and by the Zheng-Xiao-Hang-Qi project of Dongguan Polytechnic (ZHENG201608).

\section{References}

[1] Deng Xiaoheng, Luo Jie. Cooperative channel allocation and scheduling in multi-interface wireless mesh networks. Peer-to-Peer Networking and Applications, 11, pp. 1-12, 2017.

[2] Huang Xueqing, Ansari Nirwan. Resource Exchange in Smart Grid Connected Cooperative Cognitive Radio Networks. IEEE Transactions on Vehicular Technology, 7, pp. 6291-6298, 2017.

[3]Zhong, Yinzhen; Jiang, Wuxue; Ji, Shanshan. The Optimization of Distributed Cloud Computing Network Routing Model Based on MDA. Advances in Comptuer Science Research, 68, pp. 86-92, 2016. 
[4] Liang Wei , Ng Soon Xin, Hanzo Lajos. Cooperative Overlay Spectrum Access in Cognitive Radio Networks. IEEE Communications Surveys and Tutorials, 3, pp. 1924-1944, 2017.

[5] Paul Rajib, Pak Wooguil, Choi Young-June. Selectively triggered cooperative sensing in cognitive radio networks, IET Communications, 15, pp. 2720-2728, 2014.

[6] Zhu Lei, Yao Changhua, Wang Lei. The Optimization of Combination Scheme in Cooperative Spectrum Sensing Based on the Practical Reporting Frame Format. Wireless Personal Communications, 1, pp. 1-11, 2018. 